IFT/04/08

\title{
The impact of bound states on similarity renormalization group transformations
}

\author{
Stanisław D. Głazek* \\ Institute of Theoretical Physics, University of Warsaw, Poland \\ Robert J. Perry \\ Department of Physics, The Ohio State University, Columbus, OH 43210
}

(Dated: Draft of March 19, 2008)

\begin{abstract}
We study a simple class of unitary renormalization group (RG) transformations governed by a parameter $f$ in the range $[0,1]$. For $f=0$, the transformation is one introduced by Wegner in condensed matter physics, and for $f=1$ it is a simpler transformation that is being used in nuclear theory. The transformation with $f=0$ diagonalizes the Hamiltonian but in the transformations with $f$ near 1 divergent couplings arise as bound state thresholds emerge. To illustrate and diagnose this behavior, we numerically study Hamiltonian flows in two simple models with bound states: one with asymptotic freedom and a related one with a limit cycle. The $f=0$ transformation places bound-state eigenvalues on the diagonal at their natural scale, after which the bound states decouple from the dynamics at much smaller momentum scales. At the other extreme, the $f=1$ transformation tries to move bound-state eigenvalues to the part of the diagonal corresponding to the lowest momentum scales available and inevitably diverges when this scale is taken to zero. Intermediate values of $f$ cause intermediate shifts of bound state eigenvalues down the diagonal and produce increasingly large coupling constants to do this. In discrete models, there is a critical value, $f_{c}$, below which bound state eigenvalues appear at their natural scale and the entire flow to the diagonal is well-behaved. We analyze the shift mechanism analytically in a $3 \times 3$ matrix model, which displays the essense of this RG behavior and we compute $f_{c}$ for this model.
\end{abstract}

*Electronic address: stglazek@fuw.edu.pl

$\dagger$ Electronic address: perry.6@osu.edu 


\section{INTRODUCTION}

Wilsonian renormalization group transformations typically eliminate (integrate out) degrees of freedom whose energy is much higher than those of interest, replacing them with effective scale-dependent interactions. Such transformations allow one to tune an effective theory resolution, focusing on essential degrees of freedom and interactions at any scale of interest. Anticipated by Kadanoff's block spin transformation [1], early transformations explicitly reduced the number of degrees of freedom by lowering cutoffs on energy [2, 3]. Głazek and Wilson introduced a similarity renormalization group (SRG) procedure which instead uses transformations that do not remove any degrees of freedom but eliminate couplings between disparate energy scales [4, 5, 6]. After regularization and identification of necessary counterterms, the SRG procedure eventually produces a renormalized, band-diagonal matrix representation of the Hamiltonian. Independently, Wegner introduced non-perturbative differential flow equations that unitarily transform Hamiltonian matrices to the band-diagonal form [7, 8], and Wegner's flow equations can be employed in the SRG procedure.

A simplified version of Wegner's transformation [9] has been successfully applied to a number of nuclear few-body problems [10, 11]. Nuclear many-body calculations are plagued by strong nucleon-nucleon correlations due to a hard core and strong short-range tensor force, so perturbative and variational methods converge poorly. The new simple transformation produces universal nucleon-nucleon interactions with drastically improved perturbative and variational behavior. It has also been shown that one can apply SRG with Wegner's flow (or a suitably altered flow) for studying the connection between asymptotic freedom and limit cycles [12] and it has been suggested that an infrared limit cycle may exist in QCD [13]. These examples indicate that convergence properties of SRG transformations are relevant to the theory of particles over a broad range of energies.

We find that convergence properties of the simple transformation are worse than those of Wegner's transformation. The simple transformation tends to diverge whenever the SRG parameter approaches the momentum scale at which a bound state is formed in the theory. This effect may present no problem for applications in low-energy nuclear physics as long as the SRG parameter stays larger than the momentum scales at which formation of bound states occurs. However, such fortunate conditions may not be available if the Efimov effect [14, 15, 16] in the three-nucleon problem [17, 18, 19, 20] cannot be avoided. The 
Efimov effect shows up as a limit cycle. We will see that Wegner's transformation is capable of resolving limit cycle behavior, while the simple transformation diverges as the first high-energy cycle is resolved.

Transform a Hamiltonian $H(0)$ using a unitary operator $U(s)$,

$$
H(s)=U(s) H(0) U^{\dagger}(s)
$$

where $s$ is the SRG flow parameter. We want to choose $U(s)$ so that $H$ is diagonalized as $s \rightarrow \infty$ (band-diagonalized for finite $s$ ). We choose $s=0$ for the initial value, so $H(0)$ can be thought of as an input bare regularized Hamiltonian with all required and properly adjusted counterterms (such Hamiltonians are established using the same SRG procedure but this aspect is not in our focus here).

Taking derivatives of both sides of Eq. (1), we see that $H(s)$ evolves according to

$$
\frac{d H(s)}{d s}=[\eta(s), H(s)]
$$

with

$$
\eta(s)=\frac{d U(s)}{d s} U^{\dagger}(s)=-\eta^{\dagger}(s) .
$$

Choosing $\eta(s)$ specifies the transformation. We study transformations that mix two simple choices,

$$
\eta(s)=[D(s), H(s)]
$$

where $D(s)$ is the diagonal part of $H(s)$ in momentum representation, and

$$
\eta(s)=[T, H(s)]
$$

where $T$ is a fixed matrix, here chosen to be the kinetic energy. If one considers $T$ to be an arbitrary $H_{0}$ that has a known spectrum, the interaction is the remaining part, $H_{I}=H-H_{0}$.

Using $D$ in $\eta$ was Wegner's initial choice and this transformation has been studied extensively [8]. The use of $T$ was explored perturbatively [9] and then shown to effectively decouple low- and high-momentum scales in a universal characterization of the nucleonnucleon interaction [10, 11]. Universality in effective nucleon-nucleon interactions was discovered earlier [21, 22] using the same transformation Wilson used in his initial numerical RG calculations [2].

We will see that using $T$ instead of $D$ produces singularities starting at bound state thresholds and limits how far the transformation can be run. But we stress that if it is not 
run too far, for any phenomenologically tuned nucleon-nucleon interaction $H(0)$ the lowenergy part of $H(s)$ is nearly universal. This means that all infrared-constrained nucleonnucleon interactions with bare cutoffs at or even well above $500 \mathrm{MeV}$ collapse onto a nearly universal infrared Hamiltonian after the SRG parameter, $\lambda=1 / \sqrt{s}$, playing the role of effective cutoff, is evolved to well below the bare cutoff. These evolved potentials disagree only on the high-energy part of $H(s)$, which is not constrained by low-energy physics [11].

Introducing a parameter $f$ which takes values in the range $[0,1]$, we define

$$
G_{f}(s)=f T+(1-f) D_{f}(s)
$$

and write

$$
\begin{aligned}
\frac{d}{d s} H_{f}(s) & =\left[F\left\{H_{f}(s)\right\}, H_{f}(s)\right], \\
H_{f}(0) & =H
\end{aligned}
$$

where $H$ is an initial Hamiltonian matrix and the SRG generator matrix takes the form

$$
F\left\{H_{f}(s)\right\}=\left[G_{f}(s), H_{f}(s)\right]
$$

and thus defines $\eta$ that depends on $f$ and interpolates between the two cases from Eqs. (4) and (5) when $f$ varies from 0 to 1 . For explicit calculations one uses a basis in which $T$ is diagonal since it is in this representation that $H(s)$ is driven towards band-diagonal form.

The generator $F\left\{H_{f}(s)\right\}$ is guaranteed to bring the Hamiltonian to diagonal if some sufficient and easily verifiable conditions are satisfied. Such conditions will be discussed after we introduce all details that count in the derivation, in the context of two examples of basic interest in physics.

Namely, we use the family of generators $F\left\{H_{f}(s)\right\}$ to evolve Hamiltonian matrices that exhibit asymptotic freedom and limit cycle behavior. The asymptotically free matrix model is easily derived from the nonrelativstic Schrödinger equation in two dimensions with a deltafunction potential. Isolate the angular momentum zero states and discretize the momentum so that $p \rightarrow b^{n} p_{0}$ with $b>1$; include the appropriate weights to reproduce the momentum representation bound-state integral equation in the limits where $n$ is allowed to be any integer and $b \rightarrow 1$. Introduce cutoffs so that $M \leq n \leq N$ and you have the matrix we use to illustrate asymptotic freedom ( $M$ is a large negative and $N$ is a large positive integer number). The limit cycle model is obtained by adding an imaginary part to the same 
asymptotically free model Hamiltonian. The operator $T$ can be replaced by any Hermitian operator one wants to use and the methods we employ in this study can still be applied.

\section{DETAILS OF EQUATIONS}

The equations for a fixed value of $f$ contain the diagonal matrix $G_{f}(s)$ given in Eq. (6) that contains the diagonal part of $H_{f}(s)$. The Hamiltonian matrix is split into its diagonal and off-diagonal parts at every value of $s$,

$$
H_{f}(s)=D_{f}(s)+V_{f}(s)
$$

This splitting implies also

$$
\begin{aligned}
H_{f}(s) & =G_{f}(s)+\left[H_{f}(s)-G_{f}(s)\right] \\
& =G_{f}(s)+f\left[D_{f}(s)-T\right]+V_{f}(s) .
\end{aligned}
$$

This means that the diagonal part of the interaction is included in $D_{f}(s)$. The important point is that only $V_{f}(s)$ has non-zero off-diagonal matrix elements. Diagonal matrix elements of $V_{f}(s)$ are zero. $T$ and $D_{f}(s)$ are diagonal and we use an abbreviated notation for their matrix elements: $T_{m n}=T_{m} \delta_{m n}, D_{f m n}(s)=D_{m} \delta_{m n}$, and $G_{f m n}(s)=G_{m} \delta_{m n}$. Our abbreviated notation for interaction matrix elements is $V_{f m n}(s)=\left(1-\delta_{m n}\right) V_{m n}$, where $V$ is the full interaction part in the matrix $H_{f}(s)=T+V$.

The SRG Eq. (7) implies

$$
\begin{aligned}
\frac{d}{d s} D_{n} & =2 \sum_{k}\left(G_{n}-G_{k}\right) V_{n k} V_{k n}, \\
\frac{d}{d s} V_{m \neq n} & =-\left(G_{m}-G_{n}\right)\left(D_{m}-D_{n}\right) V_{m n}+\sum_{m \neq k \neq n}\left(G_{m}+G_{n}-2 G_{k}\right) V_{m k} V_{k n} .
\end{aligned}
$$

We solve these equations numerically, starting from

$$
\begin{aligned}
& H_{f}(0)_{m n}=\sqrt{E_{m} E_{n}}\left[\delta_{m n}-g-i h \operatorname{sgn}(m-n)\right], \\
& E_{n}=b^{n} \\
& M \leq n \leq N .
\end{aligned}
$$

In the case of the model with asymptotic freedom, the coupling constant $h$ is set to 0 , the Hamiltonian matrix is real and Eqs. (13) and (14) display all relevant formulae. In 
the case of the model with a limit cycle, the Hamiltonian contains an imaginary part and the equations we use to compute the Hamiltonian flow numerically involve the imaginary components,

$$
H_{f}(s)_{m n}=r_{m n}+i c_{m n}
$$

where $r$ is a real symmetric matrix and $c$ is a real antisymmetric matrix. Thus, $D_{m}=r_{m m}$. With this notation, the SRG equations we solve are

$$
\begin{aligned}
\frac{d}{d s} D_{n} & =2 \sum_{k}\left(G_{n}-G_{k}\right)\left(r_{n k}^{2}+c_{n k}^{2}\right) \\
\frac{d}{d s} r_{m \neq n} & =-\left(G_{m}-G_{n}\right)\left(D_{m}-D_{n}\right) r_{m n}+\sum_{m \neq k \neq n}\left(G_{m}+G_{n}-2 G_{k}\right)\left(r_{m k} r_{n k}+c_{n k} c_{m k}\right) \\
\frac{d}{d s} c_{m n} & =-\left(G_{m}-G_{n}\right)\left(D_{m}-D_{n}\right) c_{m n}+\sum_{m \neq k \neq n}\left(G_{m}+G_{n}-2 G_{k}\right)\left(c_{m k} r_{n k}-c_{n k} r_{m k}\right)
\end{aligned}
$$

We will show that to a good approximation $H_{f}(s)$ retains the form of $H_{f}(0)$ for matrix elements between states with kinetic energies well below the SRG parameter $\lambda=1 / \sqrt{s}$. The dominant change that occurs in these matrix elements is that $g$ is replaced by $g_{f}(s)$. This feature emerges in numerical calculations. We also analytically derive this result in the limit of large $b$ in the next section.

Once it is established through numerically calculated non-perturbative evolution of the whole Hamiltonian that its evolution can be reduced to the evolution of the coupling constant $g_{f}(s)$, we focus discussion on the evolution of the coupling and its dependence on $f$. The coupling constant is defined as

$$
g_{f}(s)=1-H_{f}(s)_{M M} / E_{M}
$$

where $E_{M}$ is the smallest energy in the matrix representation of the theory (smallest allowed eigenvalue of $T$ ) and $H_{f}(s)_{M M}$ is the smallest energy diagonal matrix element of $H_{f}(s)$, the infrared corner of the Hamiltonian. We can choose any diagonal or off-diagonal matrix element near the infrared corner of the Hamiltonian matrix to define the same coupling constant and no significant changes result until this element begins to freeze at its asymptotic limit as $s \rightarrow \infty$. Each matrix element follows a universal trajectory until it freezes at its $s \rightarrow \infty$ value. Only the lowest diagonal element displays the full evolution of the diagonal. 
When $G$ differs from $D$, i.e., $f>0$, we show that the SRG transformation does not necessarily bring the Hamiltonian matrix to diagonal form as $s \rightarrow \infty$. However, there is a sufficient condition for cases with $f>0$ that will be derived in Section IV. Namely,

$$
\frac{d V}{d T}>-1
$$

where the derivative means the rate of change of matrix elements of $V$ along the diagonal in units of rate of change of eigenvalues of $T$ along the diagonal. This condition could be violated when bound-state (negative) eigenvalues appear on the diagonal among positive eigenvalues. To study how the SRG transformation behaves depending on the choice of $f$ in the presenece of bound states, and in particular what happens in the case $f=1$ that is useful in nuclear physics, one needs to see what happens in the generic models when $f$ deviates from 0 and reaches 1 . Since the sufficient condition could be violated when the SRG parameter $\lambda$ passes the momentum scale of binding, one should find out how $H_{f}(s)$ behaves around $s$ corresponding to this region.

In the next section we discuss results of calculations of $H_{f}(s)$. We show that Wegner's transformation $(f=0)$ encounters no difficulty and places bound-state eigenvalues on the diagonal when $\lambda$ approaches the appropriate bound-state scale from above. The $f=1$ transformation breaks down as the effective cutoff approaches a bound-state scale. In fact, the transformation moves the bound-state eigenvalue to the infrared corner of the Hamiltonian. When the minimal momentum scale is much less than the bound-state momentum scale, the $f=1$ transformation forces off-diagonal matrix elements to diverge in order to move the bound-state eigenvalue to such a significantly wrong scale.

For values of $f$ between these two extremes the transformation puts the bound-state eigenvalue on the diagonal at some scale between the natural one and the infrared cutoff. However, for $f$ approaching 1, the amount of shift approaches the maximal possible and correspondingly large couplings must be generated on the way. For $f=1$, the transformation becomes numerically unstable near the bound-state scale when the infrared cutoff tends to zero.

In discrete matrix notation, one can ask how large a value of $f>0$ causes the first shift of the bound-state eigenvalue down the diagonal, just by one free energy level in comparison to the Wegner case $(f=0)$. This value of $f$ will be called critical, and denoted by $f_{c}$. It can be computed numerically to high accuracy. 


\section{NUMERICAL RESULTS: ASYMPTOTIC FREEDOM}

The characteristic SRG behavior displayed by the full matrix $H_{f}(s)$ is difficult to grasp (in our typical cases, the matrix has about 2000 matrix elements, each a function of $s$ that ranges from 0 to $\infty$ ) without studying a number of cases. Such studies involve large amounts of data. Fortunately, the overall result of such studies is that the essence of what happens for large matrices can be explained in a simple way using just one running coupling constant. Most interestingly, the characteristic behavior of this one running coupling constant in large matrices can be explained using much smaller matrices, and a matrix that is only $3 \times 3$ in size will be sufficient. These two facts guide the way we present and discuss our results.

Our goal is to describe the most important qualitative features of the Hamiltonian evolving from its initial form of the type $\sqrt{E_{m} E_{n}}\left(\delta_{m n}-g\right)$ to complete diagonalization. We need to cover a large range of scales and make important features of $V$ at all scales visible simultaneously. Taking our cue from the initial Hamiltonian and experience gathered in observing many examples of the SRG flows, we display what we will call the scaled interaction, $\mathcal{V}_{m n}=V_{m n}(s) / \sqrt{E_{m} E_{n}}$. Matrix elements of the scaled interaction are all of $\mathcal{O}(1)$ or decay to 0 during the entire process of diagonalization when Wegner's transformation is used $(f=0)$. When $f=1$ however, matrix elements of $\mathcal{V}$ diverge as the bound-state threshold is reached (see below).

Throughout its evolution, the scaled interaction matrix can be approximated by $\mathcal{V}_{m n} \sim$ $-g_{f}(s)+$ corrections for subscripts $m$ and $n$ below the transition region, in which the subscripts $m$ and $n$ take values $k$ such that $s E_{k}^{2} \sim 1$. Well above the transition region the Hamiltonian matrix is diagonalized. As $s$ increases, $g_{f}(s)$ increases to a maximum, at which point the bound-state negative eigenvalue emerges on the diagonal or a process of shifting the bound-state eigenvalue down the diagonal begins.

We start with the model case of asymptotic freedom (a discretized $\delta$-function in two dimensions), for which $h$ in eq. (15) is zero and the single bound-state eigenvalue determines $g$ through dimensional transmutation. In the notation of Section II $c_{m n}=0$ and $r_{m n}(s=$ $0)=\sqrt{E_{m} E_{n}}\left(\delta_{m n}-g\right)$. We consider $g=0.0400022797581725654$. (This particular value of $g$ is not significant; it is found from the condition that for $h=0, b=4, N=16$, $M=-25$, the bound-state eigenvalue is with high accuracy the same as one of the boundstate eigenvalues in another case: one with a limit cycle for $g=0$ and $h=\tan \pi / 50$, and 
eigenvalue $E \sim-7.64447910^{-6}$, see [12].)

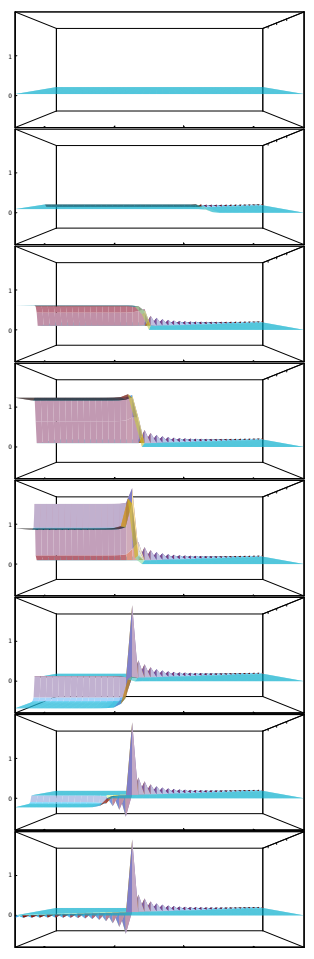

FIG. 1: SRG evolution of $H=T+V$ with $\lambda$ for $f=0$. Successive frames correspond to entries in Table I. The horizontal axes display subscripts $i$ and $j$ (running from $M$ to $N$, counting from left to right and from front to back) of the matrix $-V$ and the vertical axis displays corresponding matrix elements $-V_{i j}$ divided by $\sqrt{E_{i} E_{j}}$. See text for details.

In Fig. 1, we display frames from a movie of the evolving interaction, $V(s)$, in the Hamiltonian $H=T+V$, using Wegner's transformation, $f=0$. We show $-\mathcal{V}_{m n}$. Since small-energy matrix elements of $\mathcal{V}$ equal the negative of the coupling constant, we need to display $-\mathcal{V}$ instead of $\mathcal{V}$ itself in order to directly show how well the one coupling constant $g_{f=0}(s)$ approximates the evolution of $\mathcal{V}$ below the transition region and at the same time show how the coupling constant itself evolves. With these rescaling and display conventions, $T$ is the identity matrix. To get $H_{m n} / \sqrt{E_{m} E_{n}}$, simply take the negative of the displayed values of $-\mathcal{V}$ and add peaks of height one along the diagonal in each frame. Details concerning the frames shown in Fig. 1 are listed in Table 1 .

The negative of the scaled interaction is initially a featureless plane (frame 1), its size fixed at about 0.04 , just above zero because it is a negative of a momentum representation 
TABLE I: SRG parameters $g$ and $\lambda$ for frames shown in Fig. 1, numbered from the top to bottom. In this example, $g=0.040002, h=0, b=4, M=-25, N=16$, and all displayed numbers are rounded to 6 decimal places.

\begin{tabular}{llc}
\hline \hline frame & $\ln (\lambda) / \ln b$ & $g(\lambda)$ \\
\hline 1 (top) & 22.780321 & 0.040002 \\
2 & 2.766096 & 0.092055 \\
3 & -6.864809 & 0.600768 \\
4 & -8.369638 & 1.234710 \\
5 & -8.570281 & 0.891475 \\
6 & -9.071891 & -0.680443 \\
7 & -12.282193 & -0.226083 \\
8 (bottom) & -27.330482 & -0.060769 \\
\hline \hline
\end{tabular}

of an atractive delta-function in position representation with the initial value of the coupling constant $g \sim 0.04$. As $s$ increases from 0 , the plane drops to zero at highest energies, creating a cliff (frame 2) between the low and high energy parts of the matrix (transition region). This cliff runs along a single row and column that meet on the point along the diagonal at which newly decoupled eigenvalues are emerging. The cliff moves towards lower energies and grows in height as $s$ increases (frame 3), showing evolution of the transition region between a flat plane of zeroed high-energy off-diagonal matrix elements of $\mathcal{V}$ and a low-energy plateau that is rising higher as $s$ increases (that this plateau rises means that the coupling constant increases and the potential itself becomes more negative). The positive peaks left along the high-energy diagonal decouple from the rest of the matrix when the ridge moves past and they are basically left at the height of the low-energy rising plane at the decoupling point.

This process continues, with the low-energy part of the matrix rising and high-energy eigenvalues being left in isolation on the diagonal as the growing cliff separating low and high energy portions of the Hamiltonian moves towards the infrared corner of the matrix and the off-diagonal high-energy part of the matrix settles to zero.

We display a frame in which many high-energy eigenvalues are in place and the lowenergy plateau has barely fallen from its maximum height (frame 4), at a point where a bound state is going to emerge on the diagonal of $H(s)$. Remember that what we are 
showing is $-\mathcal{V}$, which must be multiplied by $\sqrt{E_{m} E_{n}}$ and subtracted from $T$ to produce $H(s)$. Two important things happen: the low-energy plane is high enough to cancel $T$ and produce a negative eigenvalue on the diagonal, and as this bound-state eigenvalue is left on the diagonal the low-energy plane reverses its motion (frames 4 and 5) and drops rapidly to negative values (frame 6, the coupling constant becomes negative and the whole interaction $\sim g_{f}(s)$ becomes positive, i.e., repulsive).

After the bound-state threshold is crossed, $V$ adds to the eigenvalues of $T$, while up to this point it had subtracted from these eigenvalues. After quickly reaching its deepest level, the low-energy plane gradually rises to zero (frames 7 and 8), leaving a sequence of eigenvalues that smoothly emerge from this flow one after another toward the infrared corner.

The only violent changes in these scaled variables appear around the point where the bound state emerges. This is where the coupling that characterizes the evolution of the lowenergy part of the matrix grows to its maximum value, before dropping rapidly to negative values.

It is clear that the evolution of the entire matrix $\mathcal{V}$ is well-described by the evolution of $g_{f}(s)$ in the case of $f=0$. The same is true in all other cases we consider. Therefore, we will present only the functions $g_{f}(s)$ in all these cases rather than frames from many movies.

The behavior of coupling constants in the case of asymptotic freedom for various values of $f$ is shown in Fig. 2. In the $f=1$ case, evolution of $H(s)$ is nearly identical to the $f=0$ evolution until the bound state begins to emerge. The low-energy plane in $\mathcal{V}$ moves up to produce the bound-state eigenvalue, but the bound state does not decouple from further evolution and the low-energy plane simply continues to grow apparently indefinitely and our numerical calculations fail to converge.

The $f=1$ transformation becomes singular because it tries to move the bound-state eigenvalue to the lowest-energy diagonal matrix element of the Hamiltonian, as we discuss below and explain in greater detail in the next section. This means that the bound-state wave function is being forced to include only disparately small momenta. As a result, the interactions are forced to grow to maintain observables at their true values. If the evolution is halted before the bound-state emerges, the pathological rise of interaction terms does not occur yet.

One can see in Fig. 2 not only that the larger $f$ the larger the maximal value of the coupling constant $g_{f}$ at the corresponding value of the argument $\ln \lambda_{f} / \ln b=t_{f}$, but also 


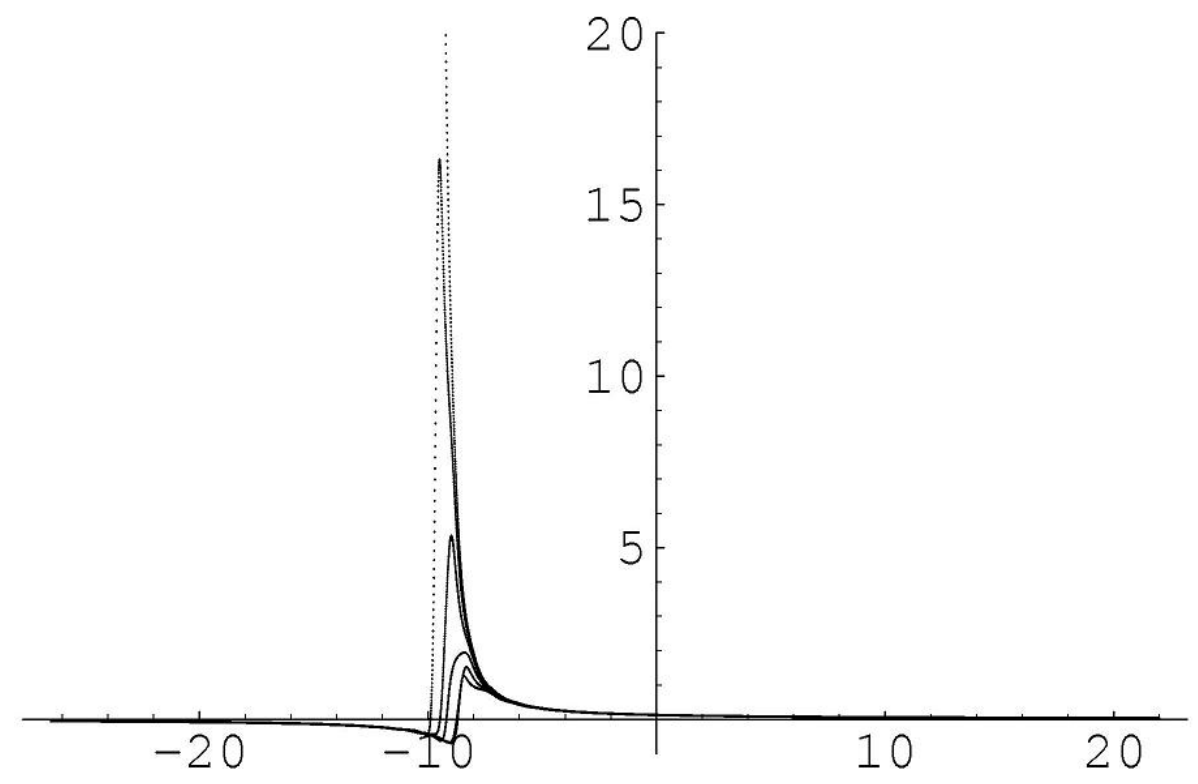

FIG. 2: The coupling constants $g_{f}$ in the case of asymptotic freedom, plotted as a function of $\ln \lambda / \ln b$ (instead of $s=1 / \lambda^{2}$ ) for 6 values of $f: f=0$ (Wegner), $f=0.2,0.5,0.75,0.9$, and 1. The correspondence between a curve and $f$ is such that the curves for larger $f$ reach higher and for $f=1$ the corresponding curve apparently shoots to infinity around $\lambda \sim\left|E_{\text {boundstate }}\right|$. The ultraviolet cutoff is at $b^{16}$, and $b=4$.

that simultaneously the argument $t_{f}$ where the maximum of $g_{f}$ is reached decreases when $f$ increases. In fact, when $f$ increases sufficiently to cause a shift of the bound-state eigenvalue by one state down the diagonal in comparison to the case $f=0$, the maximal coupling constant must increase by factor $b$, when $f$ increases sufficiently to shift the bound-state eigenvalue by two states down the diagonal, the maximal coupling constant must increase by factor $b^{2}$, and so on. Since Fig. 2 concerns the case with $b=4$, the maximal values of the coupling are about 4 and then 16 when the bound-state eigenvalue is shifted by one and two states down the diagonal, respectively. The next shift requires $g_{f} \sim 64$ and this leads to the coupling that grows out of proportion in Fig. 2. When $f=1$, the shift occurs to the minimal possible value $t_{1}=M$, and this requires that $g_{1}$ reaches $b^{|M|} E_{\text {boundstate }} \sim b^{25-9}=4^{16}$ in the case illustrated in Fig. 2, With typical machine limitations, numerical calculations are expected to fail below the scale of binding if one uses the generator $F\{H(s)\}$ with $G$ in which $f=1$. 


\section{CONVERGENCE PROBLEMS AND THE $3 \times 3$ MATRIX}

In this section, we explain the phenomenon of rise of the coupling constant when the SRG parameter approaches the scale of binding in the asymptotically free model. After that we will discuss what happens in the model with limit cycle.

We reduce the asymptotically free model to a $3 \times 3$ matrix, allowing $b$ to become arbitrarily large (i.e., there are only three, strongly coupled degrees of freedom of drastically different momentum scales), and show an analytic analysis that explains the behavior observed in the full SRG calculation for the $3 \times 3$ matrix. The $3 \times 3$ matrix model explains the mechanism that is also at work in large matrices in our models with asymptotic freedom or limit cycle.

In the $3 \times 3$ case, there is a low-energy effective coupling that evolves smoothly as the highest energy scale is decoupled by the SRG transformation. At a characteristic "time" $s$, a $2 \times 2$ low-energy effective Hamiltonian emerges, characterized by $g_{f}(s)$, and this coupling exceeds some critical value when a bound state emerges. For $f=f_{c}$, the sign of the dominant term driving further off-diagonal evolution changes, and $f_{c}$ can be computed analytically in the simple $3 \times 3$ example. Above $f_{c}$ the remaining off-diagonal matrix element is forced to diverge as a power of $b$ to force the low-energy diagonal to accommodate an eigenvalue of the wrong magnitude (wrong in the sense that it is much larger in size than the corresponding eigenvalue of $T$ ). The $b \rightarrow \infty$ limit of the full $3 \times 3$ matrix evolution can be analyzed analytically, and this is how we explain the mechanism at work in the full SRG evolution.

Before we analyze the drastically simplified $3 \times 3$ matrix truncation of the asymptotic freedom model, we recall Wegner's demonstration [7] that his transformation always diagonalizes Hamiltonians, and we use similar reasoning to explain why a simpler transformation might fail to converge.

For any similarity transformation, $\operatorname{Tr}\left(H^{2}\right)$ is independent of $s$. Separating diagonal and off-diagonal contributions to this trace, one finds

$$
\frac{d}{d s} \sum_{m} H_{m m}^{2}=-\frac{d}{d s} \sum_{m \neq n}\left|H_{m n}\right|^{2} .
$$

If the magnitudes of diagonal matrix elements increase, the magnitudes of off-diagonal matrix 
elements must decrease. Using eq. (13) for the evolution of the diagonal matrix elements,

$$
\begin{aligned}
\frac{d}{d s} \sum_{m} H_{m m}^{2} & =4 \sum_{m n} D_{m}\left(G_{m}-G_{n}\right)\left|V_{m n}\right|^{2} \\
& =2 \sum_{m n}\left(D_{m}-D_{n}\right)\left(G_{m}-G_{n}\right)\left|V_{m n}\right|^{2} .
\end{aligned}
$$

For Wegner's transformation, $G_{m}=D_{m}$, so we have

$$
\frac{d}{d s} \sum_{m} H_{m m}^{2}=2 \sum_{m n}\left(D_{m}-D_{n}\right)^{2}\left|V_{m n}\right|^{2} .
$$

No term in this sum can be negative, so the only way Wegner's transformation can stop driving off-diagonal matrix elements to zero is if degeneracies appear on the diagonal. In this case, the matrix is driven to block diagonal form with diagonal degeneracies in any non-diagonalized blocks.

In general, from Eq. (26) we see that negative terms cannot appear on the right-hand-side when all differences $D_{m}-D_{n}$ and $G_{m}-G_{n}$ always satisfy the condition

$$
\left(G_{m}-G_{n}\right)\left(D_{m}-D_{n}\right) \geq 0
$$

Introducing $(\Delta T)_{m n}=T_{m}-T_{n}$ and $(\Delta V)_{m n}=V_{m m}-V_{n n}$, one obtains for every pair of diagonal elements number $m$ and $n$,

$$
[f \Delta T+(1-f)(\Delta T+\Delta V)](\Delta T+\Delta V) \geq 0
$$

which, by dividing by $\Delta T>0$ for $m>n$, implies that $v=\Delta V / \Delta T$ must satisfy the condition

$$
[f+(1-f)(1+v)](1+v) \geq 0
$$

This condition implies for $f \in[0,1]$ that either $v \leq \frac{1}{f-1}$ or $v \geq-1$ and only for $f=0$ (Wegner's generator) these two regions can join while for $f>0$ they are always disjoint. Instead of differences for arbitrary $m$ and $n$, it is sufficient to consider differences with $m=n+1$, since all differences can be built from the differences between neighboring entries on the diagonal. Then, in the limit of continuous energy variable, the limit of $\Delta T \rightarrow 0$ produces the condition

$$
\frac{d V}{d T} \leq \frac{1}{f-1} \quad \text { or } \quad \frac{d V}{d T} \geq-1
$$


as a sufficient one for the transformation to always bring $H$ near the diagonal (outside regions of degeneracy mentioned earlier).

When $f=1, G_{m}=T_{m}$, and we see that convergence can fail if

$$
\frac{\Delta V}{\Delta T}<-1
$$

for some momenta. $T_{m}$ increases monotonically with $m$, so we see that problems can appear if $V_{m}$ decreases rapidly with $m$ in some region. This is exactly what happens when a negative value appears on the diagonal, signaling the appearance of a bound-state threshold. The appearance of negative values on the diagonal does not guarantee that the transformation will stop driving off-diagonal matrix elements to zero, and it gives no indication that offdiagonal matrix elements will actually start to diverge, but it indicates how problems can arise.

To gain further insight, we proceed to a study of the $b \rightarrow \infty$ limit, which drastically simplifies the couplings between various scales, and for further simplicity we truncate the asymptotically free Hamiltonian model to a $3 \times 3$ matrix. We refer to the three remaining scales as high, middle and low, and we write the initial Hamiltonian as:

$$
H(0)=\left(\begin{array}{ccc}
b & 0 & 0 \\
0 & 1 & 0 \\
0 & 0 & \frac{1}{b}
\end{array}\right)-g\left(\begin{array}{ccc}
b & \sqrt{b} & 1 \\
\sqrt{b} & 1 & \frac{1}{\sqrt{b}} \\
1 & \frac{1}{\sqrt{b}} & \frac{1}{b}
\end{array}\right)
$$

where the first matrix is $T$ and the second is $V(0)$. We choose $g$ so that the single negative eigenvalue is $\mathcal{O}(1)$ and should appear in the middle of the final diagonalized matrix in the case $f=0$. These conditions imply that $0.5<g<1$ and $g$ must be more than $\mathcal{O}\left(\frac{1}{b}\right)$ away from either extreme. The three eigenvalues are $(1-g) b+\mathcal{O}(1),(1-2 g) /(1-g)+\mathcal{O}(1 / b)$ and $[(1-3 g) /(1-2 g)] / b+\mathcal{O}\left(1 / b^{2}\right)$.

We define six couplings in the running interaction,

$$
V_{f}(s)=\left(\begin{array}{ccc}
-d_{h} b & -g_{h} \sqrt{b} & -g_{m} \\
-g_{h} \sqrt{b} & -d_{m} & -\frac{g_{l}}{\sqrt{b}} \\
-g_{m} & -\frac{g_{l}}{\sqrt{b}} & -\frac{d_{l}}{b}
\end{array}\right),
$$

where the diagonal couplings $d_{h}, d_{m}$ and $d_{l}$, and the off-diagonal couplings $g_{h}, g_{m}$ and $g_{l}$ are all functions of $s$. At $s=0$ all of these couplings are equal to $g$. The scaled interaction $\mathcal{V}$ is obtained from $V_{f}(s)$ by replacement of $b$ by 1 . 
Our goal is to obtain accurate estimates for all matrix elements of $V_{f}(s)$, even though their magnitudes span many orders. We cannot allow small errors in large eigenvalues or far off-diagonal matrix elements to produce large errors in small diagonal matrix elements that should reproduce the eigenvalues of order 1 or $1 / b$, the prototypical renormalization problem. As $b \rightarrow \infty$ this problem can be analyzed analytically.

We find evolution of the coupling constants in Eq. (34) using Eqs. (13) and (14). The full set of equations for arbitrary $f$ is (dots indicate derivatives with respect to $s$ )

$$
\begin{aligned}
\dot{d}_{h} & =-2 b \gamma_{h m} g_{h}^{2}-2 \gamma_{h l} g_{m}^{2} \\
\dot{d}_{m} & =2 b^{2} \gamma_{h m} g_{h}^{2}-2 b^{-1} \gamma_{h l} g_{l}^{2} \\
\dot{d}_{l} & =2 b^{2} \gamma_{h l} g_{m}^{2}+2 \gamma_{m l} g_{l}^{2} \\
\dot{g}_{h} & =-b^{2} \gamma_{h m}\left[\left(1-d_{h}\right)-b^{-1}\left(1-d_{m}\right)\right] g_{h}-\left(\gamma_{h l}+b^{-1} \gamma_{m l}\right) g_{m} g_{l}, \\
\dot{g}_{m} & =-b^{2} \gamma_{h l}\left[\left(1-d_{h}\right)-b^{-2}\left(1-d_{l}\right)\right] g_{m}-b\left(\gamma_{h m}-b^{-1} \gamma_{m l}\right) g_{h} g_{l}, \\
\dot{g}_{l} & =b^{2}\left(\gamma_{h m}+\gamma_{h l}\right) g_{h} g_{m}-\gamma_{m l}\left[\left(1-d_{m}\right)-b^{-1}\left(1-d_{l}\right)\right] g_{l},
\end{aligned}
$$

where

$$
\begin{aligned}
\gamma_{h m} & =\gamma_{h}-\gamma_{m} / b \\
\gamma_{m l} & =\gamma_{m}-\gamma_{l} / b \\
\gamma_{h l} & =\gamma_{h}-\gamma_{l} / b^{2} \\
\gamma_{h} & =f+(1-f)\left(1-d_{h}\right), \\
\gamma_{m} & =f+(1-f)\left(1-d_{m}\right), \\
\gamma_{l} & =f+(1-f)\left(1-d_{l}\right) .
\end{aligned}
$$

\section{A. Approximate evolution of $3 \times 3$ matrix for $f=0$}

We begin by considering Wegner's transformation, $f=0$. We use Eqs. (35)-(40) and keep only the leading terms for large $b$. We will see that all couplings remain $\mathcal{O}(1)$ when $f=0$, so this analysis is fairly straightforward. The evolution has two stages: elimination of $g_{h}$ and $g_{m}$ in the first stage, and elimination of $g_{l}$ in the second stage. Terms driving the first stage of evolution are $\mathcal{O}\left(b^{2}\right)$ and govern evolution until $s$ exceeds $\mathcal{O}\left(1 / b^{2}\right)$. These terms are then exponentially suppressed by $g_{h}$ and $g_{m}$, and the second stage of evolution is 
governed by subleading terms. The leading terms are:

$$
\begin{aligned}
\frac{d}{d s} d_{h} & =-2\left(1-d_{h}\right) g_{h}^{2} b+\mathcal{O}(1), \\
\frac{d}{d s} d_{m} & =2\left(1-d_{h}\right) g_{h}^{2} b^{2}+\mathcal{O}(b), \\
\frac{d}{d s} d_{l} & =2\left(1-d_{h}\right) g_{m}^{2} b^{2}+\mathcal{O}(1), \\
\frac{d}{d s} g_{h} & =-\left(1-d_{h}\right)^{2} g_{h} b^{2}+\mathcal{O}(b), \\
\frac{d}{d s} g_{m} & =-\left(1-d_{h}\right)^{2} g_{m} b^{2}+\mathcal{O}(b), \\
\frac{d}{d s} g_{l} & =2\left(1-d_{h}\right) g_{h} g_{m} b^{2}+\mathcal{O}(b) .
\end{aligned}
$$

We see from (50) and (51) that $g_{h}=g_{m}$ during the first stage, because all couplings start at $g$. This implies that $d_{m}=d_{l}=g_{l}$ during this stage also, because the leading equations governing their evolution become identical. Since the low energy $2 \times 2$ submatrix of $\mathcal{V}$ is determined by $d_{m}, d_{l}$ and $g_{l}$, it retains its original form during the first stage of evolution, with a single coupling that can be factored from the submatrix of $\mathcal{V}$. This is one of the most important results of this analysis and it can be generalized to larger matrices.

The leading term on the right of Eq. 47) is $\mathcal{O}(b)$ rather than $\mathcal{O}\left(b^{2}\right)$, so for $s$ of $\mathcal{O}\left(1 / b^{2}\right)$, $d_{h}$ changes only by $\mathcal{O}(1 / b)$ and we can ignore this change when solving Eqs. (50) and (51), replacing $d_{h}$ with its initial value, $g$. Solving Eqs. (50) and (51) for large $b$ we obtain

$$
g_{h}(s) \approx g_{m}(s) \approx g \mathrm{e}^{-(1-g)^{2} b^{2} s} .
$$

Both of these couplings decay to zero exponentially, and for large $b$ this decay is so rapid that it can be treated as instantaneous. Inserting Eq. (53) in Eq. (47) we find,

$$
d_{h}=g-\frac{g^{2}}{(1-g) b}+\mathcal{O}\left(1 / b^{2}\right)
$$

at the end of the first stage of evolution. For a complete leading-order analysis, we need only these leading approximations for $g_{h}, g_{m}$ and $d_{h}$. The largest eigenvalue, $\left(1-d_{h}\right) b \approx$ $(1-g) b+\mathcal{O}(1)$ thus appears in the high-energy corner of the matrix. We do not need the $\mathcal{O}(1 / b)$ correction to $d_{h}$ to obtain the highest eigenvalue accurately for large $b$ and we will see that we do not need this correction to accurately compute the smaller eigenvalues either.

The leading term governing the early evolution of the remaining $2 \times 2$ submatrix is

$$
2\left(1-d_{h}\right) g_{h}^{2} b^{2} \approx 2(1-g) g^{2} b^{2} \mathrm{e}^{-2(1-g)^{2} b^{2} s} \approx \frac{g^{2}}{1-g} \delta(s)
$$


where $\delta(s)$ is defined so that $\int_{0}^{\infty} \delta(s) d s=1$. The couplings $d_{m}, d_{l}$ and $g_{l}$ instantly increase by $g^{2} /(1-g)$, so at the end of the first stage and the beginning of the second stage of evolution

$$
d_{m}(s) \approx d_{l}(s) \approx g_{l}(s) \approx \frac{g}{1-g}+\mathcal{O}(1 / b)
$$

For the range of $g$ that produces a binding energy of $\mathcal{O}(1)$, these low- and middle-energy couplings exceed 1 and negative values appear on the diagonal of $H$ at the start of the second stage of evolution.

After the initial instant of evolution, $g_{h} \approx 0, g_{m} \approx 0$ and $d_{h} \approx g$. Corrections to these approximations have no effect on the leading order of any eigenvalue. For example, the $\mathcal{O}(1 / b)$ correction to $d_{h}$ has no effect on the smallest eigenvalue, which is $\mathcal{O}(1 / b)$. Returning to the full equations, replacing $g_{h}, g_{m}$ and $d_{h}$ with these approximations, choosing an initial value of $g /(1-g)$ for $d_{m}, d_{l}$ and $g_{l}$, we find the leading-order equations that govern the second stage of evolution:

$$
\begin{aligned}
\frac{d}{d s} d_{m} & \approx-2\left(1-d_{m}\right) g_{l}^{2} \frac{1}{b} \approx 0, \\
\frac{d}{d s} d_{l} & \approx 2\left(1-d_{m}\right) g_{l}^{2}, \\
\frac{d}{d s} g_{l} & \approx-\left(1-d_{m}\right)^{2} g_{l} .
\end{aligned}
$$

When $g$, the initial coupling, is chosen so that the binding energy is $\mathcal{O}(1), 1-d_{m}<0$ at the start of the second stage and for large $b, d_{m}$ does not evolve further. This means that the negative eigenvalue, $1-d_{m}=1-g /(1-g)=(1-2 g) /(1-g)$ appears in the middle of the matrix and stays there. This leaves us with,

$$
\begin{aligned}
\frac{d}{d s} d_{l} & \approx 2 \frac{1-2 g}{1-g} g_{l}^{2}, \\
\frac{d}{d s} g_{l} & \approx-\left(\frac{1-2 g}{1-g}\right)^{2} g_{l} .
\end{aligned}
$$

From these we see that

$$
g_{l}(s) \approx \frac{g}{1-g} \mathrm{e}^{-(1-2 g)^{2} /(1-g)^{2} s}
$$

which leads as $s \rightarrow \infty$ to

$$
d_{l}(s) \rightarrow \frac{g}{1-2 g} .
$$

This means that the smallest eigenvalue, $\left(1-d_{l}\right) / b \approx[(1-3 g) /(1-2 g)] / b$, appears in the infrared corner of the matrix when $f=0$ and no couplings become unnaturally large during the evolution. 


\section{B. Approximate evolution of $3 \times 3$ matrix for $f=1$}

Next we study the transformation when $f=1$. We again use Eqs. (35)-(40) and keep only the leading terms for large $b$ for the first stage of the evolution. We will see that $g_{h}$ and $g_{m}$ again decay exponentially and all other couplings remain $\mathcal{O}(1)$ during this first stage, but when $f=1$ the coupling $g_{l}$ grows during the second-stage to $\mathcal{O}\left(b^{1 / 2}\right)$ before it is finally driven to zero and the second-stage's analysis is much more complicated because of this unnatural growth. The leading terms for $f=1$ are:

$$
\begin{aligned}
\frac{d}{d s} d_{h} & =-2 g_{h}^{2} b+\mathcal{O}(1), \\
\frac{d}{d s} d_{m} & =2 g_{h}^{2} b^{2}+\mathcal{O}(b), \\
\frac{d}{d s} d_{l} & =2 g_{m}^{2} b^{2}+\mathcal{O}(1), \\
\frac{d}{d s} g_{h} & =-\left(1-d_{h}\right) g_{h} b^{2}+\mathcal{O}(b), \\
\frac{d}{d s} g_{m} & =-\left(1-d_{h}\right) g_{m} b^{2}+\mathcal{O}(b), \\
\frac{d}{d s} g_{l} & =2 g_{h} g_{m} b^{2}+\mathcal{O}(b) .
\end{aligned}
$$

These equations govern the first stage of the SRG evolution, with subleading terms becoming important in the second stage. We again see that $g_{h}=g_{m}$ to leading order, which implies that $d_{m}=d_{l}=g_{l}$ to leading order during the first stage. The low energy $2 \times 2$ submatrix is determined by these three couplings, and it retains the form of the initial Hamiltonian for all values of $f$ during the first stage of evolution, so we can use the universal coupling governing the submatrix to characterize the transformation for all values of $f$. Solving the equations for $g_{h}$ and $g_{m}$ for large $b$, using the fact that $d_{h}=g+\mathcal{O}(1 / b)$, we obtain

$$
g_{h}(s) \approx g_{m}(s) \approx g \mathrm{e}^{-(1-g) b^{2} s}
$$

This is nearly the same result we obtained in Eq. (53) for $f=0$ but the exponent is different. Both couplings decay to zero exponentially and $d_{h}$ changes only at $\mathcal{O}(1 / b)$ because the exponent is $\mathcal{O}\left(b^{2}\right)$ while the term driving the evolution of $d_{h}$ is only $\mathcal{O}(b)$. The largest eigenvalue, $\left(1-d_{h}\right) b \approx(1-g) b$ again appears in the high-energy corner of the matrix.

The leading term governing the early evolution of the remaining $2 \times 2$ submatrix is

$$
2 g_{h}^{2} b^{2} \approx 2 g^{2} b^{2} \mathrm{e}^{-2(1-g) b^{2} s} \approx \frac{g^{2}}{1-g} \delta(s),
$$


where $\delta(s)$ is again defined so that $\int_{0}^{\infty} \delta(s) d s=1$. Once again we find that the couplings $d_{m}, d_{l}$ and $g_{l}$ instantly increase by $g^{2} /(1-g)$, to leading order, so at the beginning of the second stage of evolution

$$
d_{m}(s) \approx d_{l}(s) \approx g_{l}(s) \approx \frac{g}{1-g} .
$$

These second-stage "initial" values can differ at $\mathcal{O}(1 / b)$, but such differences do not affect the subsequent analysis when $f=0$. We will see that a full analysis when $f=1$ is sensitive to such $\mathcal{O}(1 / b)$ corrections, but we are not interested in the effects of these small corrections. We focus on how the transformation works when the simple scaling analysis breaks down. We want to understand the origin of this breakdown and we can infer its consequences without a derivation of the precise evolution of the couplings. The origin lies in the specific SRG transformation, not in the precise second-stage initial values of the coupling constants.

For the range of $g$ that produces an $\mathcal{O}(1)$ binding energy, the low- and middle-energy couplings exceed one and negative values again appear on the diagonal of $H$. However, when $f=1$ the appearance of negative values on the diagonal can signal trouble, as argued at the beginning of this section.

After the initial stage of evolution, $g_{h} \approx 0, g_{m} \approx 0$ and $d_{h} \approx g$. The subleading terms that govern the second stage of evolution in this case are:

$$
\begin{aligned}
\frac{d}{d s} d_{m} & \approx \frac{-2}{b} g_{l}^{2}, \\
\frac{d}{d s} d_{l} & \approx 2 g_{l}^{2}, \\
\frac{d}{d s} g_{l} & \approx-\left(1-d_{m}\right) g_{l}+\frac{1-d_{l}}{b} g_{l} .
\end{aligned}
$$

When the binding energy is $\mathcal{O}(1)$, at the start of the second stage $1-d_{m}<0$ in Eq. (75), so $g_{l}$ grows exponentially rather than decaying as it did when $f=0$. Eq. (73) implies that $d_{m}$ will decrease monotonically at $\mathcal{O}(1 / b)$, but before it can decrease sufficiently to reverse the growth of $g_{l}, g_{l}$ grows to $\mathcal{O}(\sqrt{b})$. Meanwhile, Eq. 74 implies that $d_{l}$ will grow monotonically and it eventually grows to $\mathcal{O}(b)$ as the bound state eigenvalue is moved from the middle of $H$ to its low-energy corner. Once $d_{m}$ decreases sufficiently and $d_{l}$ grows sufficiently, the sign of the right-hand-side of Eq. (75) changes and $g_{l}$ is driven to zero exponentially from this point.

An exact solution of these equations is readily found because the unitary evolution of the $2 \times 2$ low-energy submatrix of $H$ is simply a rotation that conserves its trace and determinant. 
Since $d_{m}$ decreases monotonically, the bound state eigenvalue cannot appear in the middle of the matrix, as it did when $f=0$. The low-energy eigenvalue that is $\mathcal{O}(1 / b)$ must end up in the middle of the matrix, and this means the precise final result of the transformation is sensitive to $\mathcal{O}(1 / b)$ corrections to the initial value of $d_{m}$, but the mechanism by which the $\mathcal{O}(1)$ eigenvalue is transferred to the $\mathcal{O}(1 / b)$ momentum corner of $H$ is not. Thus, the unitarity of the transformation combined with the orders of magnitude of the couplings $d_{m}, g_{l}$ and $d_{l}$ at the beginning of the second stage are enough to diagnose why numerical transformations of large matrices fail to converge for some transformations. Moving the bound state eigenvalue to an "unnatural" location requires growth of couplings by powers of $b$, and in large matrices additional powers of $b$ appear each time the bound state eigenvalue is moved one step down along the diagonal.

\section{The critical value $f_{c}$}

We do not provide complete details of the full evolution for arbitrary $f$ between zero and one; the critical value, $f_{c}$, is revealed at the end of the first stage of evolution. As the bound-state eigenvalue emerges on the diagonal, $f$ determines whether $g_{l}$ grows or decays exponentially.

We again use Eqs. (35)- 40 and keep only the leading terms for large $b$.

$$
\begin{aligned}
\frac{d}{d s} d_{h} & =-2\left[1-(1-f) d_{h}\right] g_{h}^{2} b+\mathcal{O}(1) \\
\frac{d}{d s} d_{m} & =2\left[1-(1-f) d_{h}\right] g_{h}^{2} b^{2}+\mathcal{O}(b) \\
\frac{d}{d s} d_{l} & =2\left[1-(1-f) d_{h}\right] g_{m}^{2} b^{2}+\mathcal{O}(1), \\
\frac{d}{d s} g_{h} & =-\left(1-d_{h}\right)\left[1-(1-f) d_{h}\right] g_{h} b^{2}+\mathcal{O}(b) \\
\frac{d}{d s} g_{m} & =-\left(1-d_{h}\right)\left[1-(1-f) d_{h}\right] g_{m} b^{2}+\mathcal{O}(b) \\
\frac{d}{d s} g_{l} & =2\left[1-(1-f) d_{h}\right] g_{h} g_{m} b^{2}+\mathcal{O}(b)
\end{aligned}
$$

As expected from the two extremes $f=0$ and $f=1$, during the first stage of evolution, $g_{h}=g_{m}$, implying that $d_{m}=d_{l}=g_{l}$. Moreover, the entire analysis of the first stage of evolution is qualitatively independent of $f$. To leading order:

$$
g_{h}(s)=g_{m}(s)=g \mathrm{e}^{-(1-g)[1-(1-f) g] b^{2} s},
$$


cf. Eqs. (53) and (70).

Once again the integrated effect of these couplings on the low-energy $2 \times 2$ submatrix is to shift the submatrix couplings to a new starting value, at $s=0_{+}=\mathcal{O}\left(1 / b^{2}\right)$,

$$
g_{l}\left(0_{+}\right)=d_{m}\left(0_{+}\right)=d_{l}\left(0_{+}\right)=\frac{g}{1-g},
$$

after which we need only examine the equation governing subsequent evolution of $g_{l}$ :

$$
\frac{d}{d s} g_{l}=-\left[1-d_{m}(s)\right]\left[1-(1-f) d_{m}(s)\right] g_{l}(s)+\mathcal{O}(1 / b) .
$$

This off-diagonal coupling grows for $d_{m}(s)>1$ if

$$
1-(1-f) \frac{g}{1-g}>0
$$

from which we determine

$$
f_{c}=2-\frac{1}{g} .
$$

This value is always between zero and one if there is a bound state with a binding energy that is $\mathcal{O}(1)$.

The evolution of larger matrices as $b \rightarrow 1$ is far more complicated than the $b \rightarrow \infty$ limit of the $3 \times 3$ matrix, but there is remarkable similarity. Any stage in the evolution of larger matrices can be modeled by a $3 \times 3$ matrix with the middle diagonal matched to the point on the diagonal where an eigenvalue is emerging. We have focused on the point where a bound-state eigenvalue appears, because in the evolution of large matrices with $b$ near 1 , it is at this point in the SRG evolution of the Hamiltonian that transformations bifurcate; those with $f<f_{c}$ leave the bound state eigenvalue on the diagonal where it appears for $f=0$, while those with $f>f_{c}$ move it down the diagonal. Transformations with $f \rightarrow 1$ misplace the bound-state eigenvalue to such small scales that they fail to numerically converge. Offdiagonal matrix elements are forced to diverge exponentially to accomplish this. There is always a tipping point, $f_{c}<1$, and at $f_{c}$ when the bound state emerges, off-diagonal matrix elements are balanced at an $\mathcal{O}(1)$ value between regions of exponential decay and exponential growth.

\section{NUMERICAL RESULTS: LIMIT CYCLE}

The limit cycle Hamiltonian of Eq. (15) with $h \neq 0$ possesses many bound states, one for each cycle. The number of eigenvalues that emerge in each cycle is fixed by $h$. Application 
of the SRG procedure in the case of a limit cycle is based on [12]. Numerical calculation in this case produces Fig. 3. One sees that the case of asymptotic freedom in the previous subsection corresponds in its behavior around the scale of binding to one cycle around the scale of binding in the limit cycle. In addition, one sees that when $f \rightarrow 1$ and the SRG generator becomes purely kinematic, the RG evolution is stuck in the range of scales corresponding to the scale of binding in the first cycle (greatest binding energy). In order to get to the next cycle and smaller binding energies, one has to introduce dynamics into the generator $[G, H]$ through the operator $G$.

Note that Fig. 3 also shows that the larger the shifts of the bound-state eigenvalues down the diagonal and the larger the corresponding coupling constant, the more sensitive the numerics are to details of the finite matrix, introducing departures from a clean cycle. These numerical effects are not fully understood but they have no bearing on the findings reported here that concern the impact of bound states on the SRG transformations.

It is now clear that in the cases where the greatest binding energy $E$ is very small, one does not encounter problems using purely kinematic $G=T$ as long as $\lambda \gg|E|$. This is our explanation of what happens in the studies in nuclear and atomic physics where the binding energies are much smaller than the cutoffs on momenta. Hadronic structure is different because there are no free quarks or gluons; all high energy eigenstates involve bound states of quarks and gluons so it is not possible to remain above a bound-state threshold.

When one proceeds to the case of a limit cycle with a geometric series of binding energies, one must insure that the largest of them is small in comparison with desired values of $\lambda$ for the generator with $G=T$ to work.

\section{CONCLUSION}

The SRG offers an alternative to traditional renormalization group transformations which discard degrees of freedom and is being developed to attack problems where traditional methods fail. These problems all involve a broad (in principle, considered infinite) range of momentum scales that are strongly coupled and are typically not amenable to perturbation theory, even if the latter is so-called renormalization-group improved. Analytic methods fail and direct numerical solutions are not possible because the space of states cannot be truncated, due to the strong coupling, and the full space is too large for current or foreseeable 
numerical storage and manipulation. In the most interesting problems one cannot even be sure what operators are needed to construct renormalizable Hamiltonians (e.g., what counterterms are required in the presence of known bare interactions).

We have not illustrated the initial steps required to find renormalizable Hamiltonians, choosing simple model problems in which the required operators are basically known (e.g., see [23]). But our calculations do illustrate methods that can make such initial steps feasible. First, as anticipated in Wilson's earliest work [24], we drastically truncate the space of states by moving from a continuum to a space of discrete states which are spaced exponentially, with a tunable spacing governed by a parameter $b$. Going from a well-defined continuum problem to a discretized problem is straightforward in the models we use, so we have paid little attention to this step; nor have we shown that with the renormalization problems we solve the continuum can be recovered with exponential convergence by letting $b \rightarrow 1$. The relevance of our calculations rests on the assumption that such a limit can be taken with control of errors and without introducing new renormalization problems. Once we identify

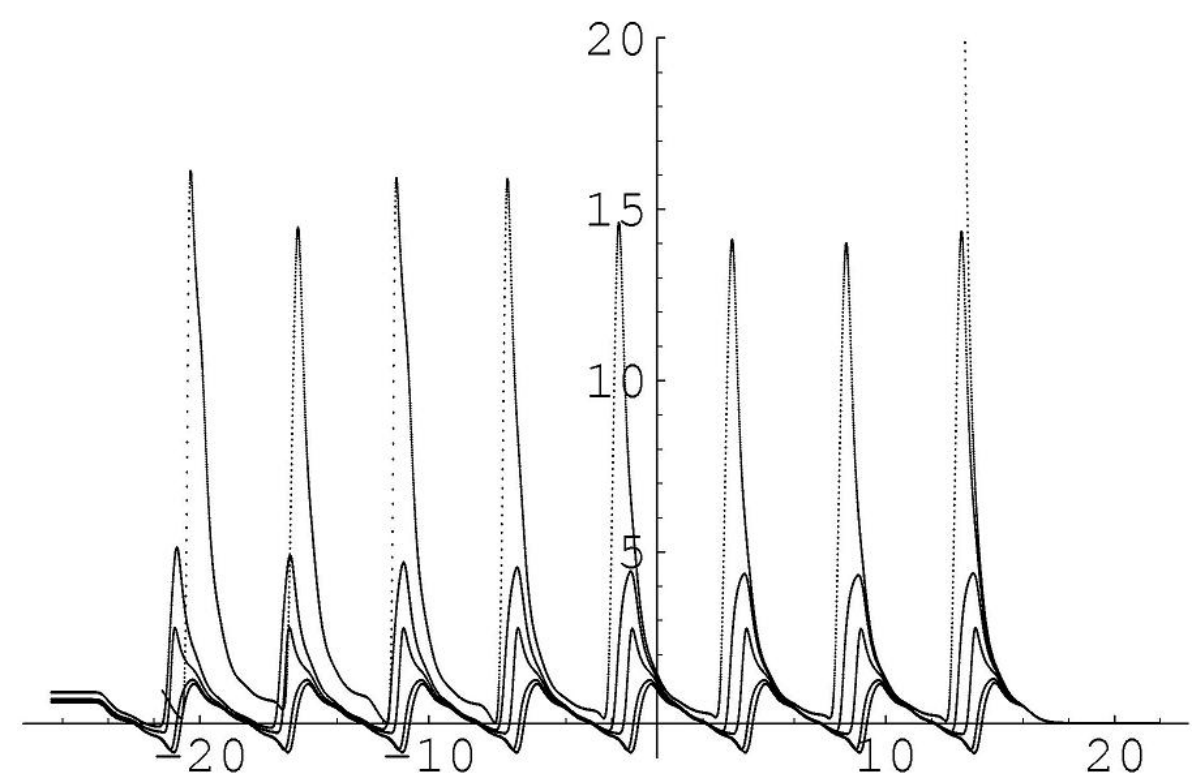

FIG. 3: The coupling constants $g_{f}(\lambda)$ in the case of a limit cycle, plotted as a function of $\ln \lambda / \ln b$ for 6 values of $f: f=0$ (Wegner), $f=0.2,0.5,0.75,0.9$, and 1 . The correspondence between a curve and $f$ is such that the curves for larger $f$ reach higher and for $f=1$ the corresponding curve apparently shoots to infinity already around $\ln \lambda / \ln b \sim 15$. The ultraviolet cutoff is at $b^{16}$ and $b=4$, see [12]. 
the operators required for renormalization with large $b$, using nonperturbative numerical calculations like those shown here, we reach what is the starting point for the calculations we present.

Attempts to control the effects of strong coupling over large numbers of momentum scales typically fail because divergences emerge. Originally, such divergences showed up in perturbation theory and gave rise to the whole renormalization program of the last century. Far more challenging are the types of divergence illustrated by our calculations, divergences that persist if one attempts to go beyond perturbation theory using perturbative renormalization schemes such as standard methods for the renormalization of quantum electrodynamics. Wilson's renormalization group improved perturbation theory avoids many of these problems in asymptotically free theories as long as cutoffs are kept sufficiently large. However, to solve problems in which strong-coupling fixed points [3] or limit cycles exist, renormalization group transformations often must be crafted on a case by case basis. What the SRG offers is a wealth of new transformations and in this paper we have focussed on some of the critical features of these new transformations.

The existence of a critical value $f=f_{c}$ in SRG transformations with generators $F\{H\}=$ $[G, H]$ and $G=f T+(1-f) D$, is demonstrated in discrete models with important features such as asymptotic freedom or a limit cycle. The case of SRG with $f=1$, or $G=T$, is always in the region $f>f_{c}$. Therefore, when bound states exist and the SRG parameter $\lambda=1 / \sqrt{s}$ approaches the scale of momenta that dominate in the formation of bound states, the strength of renormalized interactions grows. Numerical calculations of the interaction Hamiltonians become increasingly difficult due to this growth. But if it is enough to calculate effective theories with $\lambda$ much larger than the scale of binding, the generator with $f=1$ can be employed without encountering an intractable increase of interaction strength.

As long as one stays away from the bound-state momentum scale, the numerical calculations in the generic models are equally powerful for $f>f_{c}$ as they are for $f<f_{c}$. This result shows that limits on applicability of the simplest version of the SRG transformation with $f=1$ due to bound states are not as severe as one might expect provided one keeps $\lambda$ away from the scale of binding. This is important because the SRG transformations with $f=1$ are the simplest to implement in the continuum limit and in perturbative evaluation of the SRG flow of Hamiltonians.

At the same time, it is also made clear that in order to handle cases with a limit cycle, 
one has to consider $f<f_{c}$. This means that the generator must include interactions in $G$ and cannot be limited to $T$. The $f=0$ transformation advocated by Wegner [7, 8] is able to drive both of our models to diagonal form. We have not shown that this reproduces both the correct binding energy and phase shifts for the continuum problem but such a demonstration should be straightforward and is left for future work.

Finally, it should be pointed out that this article does not resolve many important issues that must be resolved to deal with confinement. These go well beyond dealing with bound states and are not encountered in our simple generic models, but confinement presents us with the problem of bound states at all cutoffs and at least in this respect the limit cycle model should provide important insights.

\section{Acknowledgments}

We thank Scott Bogner and Dick Furnstahl for useful comments. This work was supported in part by the National Science Foundation under Grant No. PHY-0653312.

[1] L. P. Kadanoff, Physica 2, 263 (1965).

[2] K. G. Wilson, Phys. Rev. D2 1438 (1970).

[3] K. G. Wilson, Rev. Mod. Phys. 47, 773 (1975).

[4] S. D. Głazek and K. G. Wilson, Phys. Rev. D 48, 5863 (1993).

[5] S. D. Głazek and K. G. Wilson, Phys. Rev. D 49, 4214 (1994).

[6] S. D. Głazek and K. G. Wilson, Phys. Rev. D 57, 3558 (1998).

[7] F. Wegner, Ann. Phys. (Leipzig) 3, 77 (1994).

[8] S. Kehrein, The Flow Equation Approach to Many-Particle Systems (Springer, 2006).

[9] S. Szpigel, R. J. Perry, in Quantum Field Theory, A 20th Century Profile, Ed. A. N. Mitra, p. $59 ;$ hep-ph/0009071.

[10] S. K. Bogner, R. J. Furnstahl and R. J. Perry, Phys. Rev. C 75 (2007) 061001(R).

[11] S.K. Bogner, R.J. Furnstahl, R.J. Perry and A. Schwenk, Phys. Lett. B 649 (2007) 488.

[12] S. D. Głazek, Phys. Rev. D 75, 025005 (2007).

[13] E. Braaten and H.-W. Hammer, Phys. Rev. Lett. 91 (2003) 102002. 
[14] L. H. Thomas, Phys. Rev. 47, 903 (1935).

[15] V. Efimov, Phys. Lett. 33B, 563 (1970).

[16] V. N. Efimov, Sov. J. Nucl. Phys. 12, 589 (1971) [Yad. Fiz. 12, 1080 (1970)].

[17] P. F. Bedaque, H.-W. Hammer, and U. van Kolck, Phys. Rev. Lett. 82, 463 (1999) arXiv:nuclth/9809025.

[18] P. F. Bedaque, H.-W. Hammer, and U. van Kolck, Nucl. Phys. A 646, 444 (1999) arXiv:nuclth/9811046].

[19] P. F. Bedaque, H.-W. Hammer, and U. van Kolck, Nucl. Phys. A 676, 357 (2000) arXiv:nuclth/9906032.

[20] R.F. Mohr, R.J. Furnstahl, R.J. Perry, K.G. Wilson and H.W. Hammer, Annals Phys. 321 225 (2006).

[21] S. K. Bogner, A. Schwenk, T. T. S. Kuo and G. E. Brown, nucl-th/0111042.

[22] S. K. Bogner, T. T. S. Kuo and A. Schwenk, Phys. Rept. 386, 1 (2003).

[23] S. D. Głazek, K. G. Wilson, Phys. Rev. B 69, 094304 (2004).

[24] K. G. Wilson, Phys. Rev. 140 B445 (1965). 